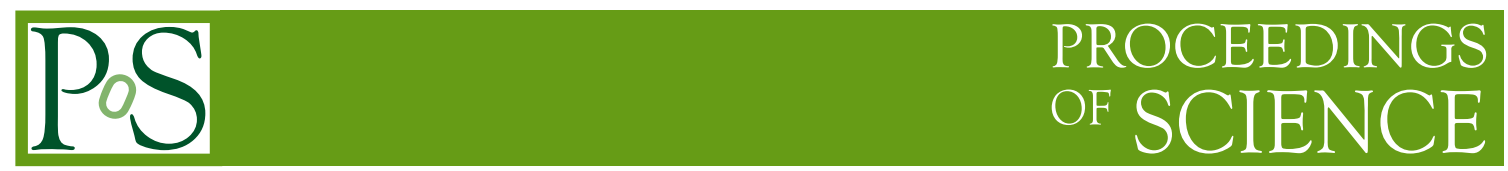

\title{
The TIBET AS+MD Project; progress report 2015
}

M. Amenomori ${ }^{1}$, X. J. Bi ${ }^{2}$, D. Chen ${ }^{3}$, T. L. Chen ${ }^{4}$, W. Y. Chen ${ }^{2}$, S. W. Cui ${ }^{5}$, Danzengluobu $^{4}$, L. K. Ding ${ }^{2}$, C. F. Feng ${ }^{6}$, Zhaoyang Feng ${ }^{2}$, Z. Y. Feng ${ }^{7}$, Q. B. Gou ${ }^{2}$, Y. Q. Guo ${ }^{2}$, H. H. He ${ }^{2}$, Z. T. He ${ }^{5}$, K. Hibino ${ }^{8}$, N. Hotta ${ }^{9}$, Haibing $\mathrm{Hu}^{4}$, H. B. $\mathrm{Hu}^{2}$, J. Huang ${ }^{2}$, H. Y. Jia ${ }^{7}$, L. Jiang ${ }^{2}$, F. Kajino ${ }^{10}$, K. Kasahara ${ }^{11}$, Y. Katayose ${ }^{12}$, C. Kato ${ }^{13}$, K. Kawata ${ }^{14}$, M. Kozai ${ }^{13}$, Labaciren $^{4}$, G. M. Le ${ }^{15}$, A. F. Li ${ }^{16,6,2}$, H. J. Li ${ }^{4}$, W. J. Li ${ }^{2,7}$, C. Liu $^{2}$, J. S. Liu' ${ }^{2}$, M. Y. Liu ${ }^{4}$, H. Lu' ${ }^{2}$, X. R. Meng ${ }^{4}$, T. Miyazaki ${ }^{13}$, K. Mizutani ${ }^{11,17}$, K. Munakata ${ }^{13}$, T. Nakajima ${ }^{13}$, Y. Nakamura ${ }^{13}$, H. Nanjo ${ }^{1}$, M. Nishizawa ${ }^{18}$, T. Niwa ${ }^{13}$, M. Ohnishi ${ }^{14}$, I. Ohta ${ }^{19}$, S. Ozawa ${ }^{11}$, X. L. Qian ${ }^{6,2}$, X. B. Qu ${ }^{20}$, T. Saito ${ }^{21}$, T. Y. Saito ${ }^{22}$, M. Sakata ${ }^{10}$, T. K. Sako ${ }^{14}$, J. Shao ${ }^{2,6}$, M. Shibata ${ }^{12}$, A. Shiomi ${ }^{23}$, T. Shirai ${ }^{8}$, H. Sugimoto ${ }^{24}$, M. Takita ${ }^{14}$, Y. H. Tan ${ }^{2}$, N. Tateyama ${ }^{8}$, S. Torii ${ }^{11}$, H. Tsuchiya ${ }^{25}$, S. Udo ${ }^{8}$, H. Wang ${ }^{2}$, H. R. Wu ${ }^{2}$, L. Xue ${ }^{6}$, Y. Yamamoto ${ }^{10}$, K. Yamauchi ${ }^{12}$, Z. Yang ${ }^{2}$, S. Yasue ${ }^{26}$, A. F. Yuan ${ }^{4}$, T. Yuda ${ }^{14}$, L. M. Zhai ${ }^{2}$, H. M. Zhang ${ }^{2}$, J. L. Zhang ${ }^{2}$, X. Y. Zhang ${ }^{6}$, Y. Zhang ${ }^{2}$, Yi Zhang ${ }^{2}$, Ying Zhang ${ }^{2}$, Zhaxisangzhu ${ }^{4}$, X. X. Zhou ${ }^{7}$ 


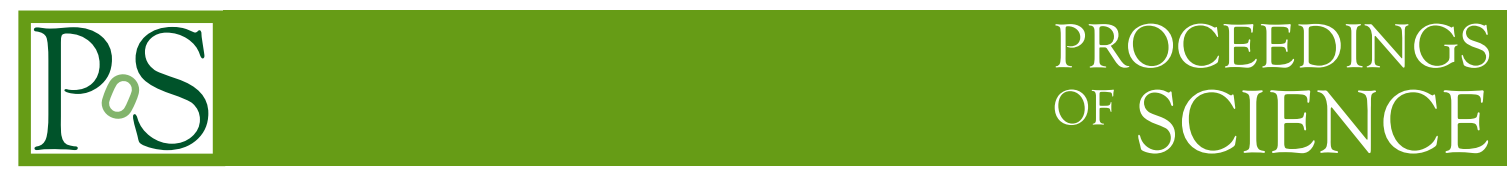

(The Tibet AS $\gamma$ Collaboration)

${ }^{1}$ Department of Physics, Hirosaki University, Hirosaki 036-8561, Japan

${ }^{2}$ Key Laboratory of Particle Astrophysics, Institute of High Energy Physics, Chinese Academy of Sciences, Beijing 100049, China

${ }^{3}$ National Astronomical Observatories, Chinese Academy of Sciences, Beijing 100012, China

${ }^{4}$ Department of Mathematics and Physics, Tibet University, Lhasa 850000, China

${ }^{5}$ Department of Physics, Hebei Normal University, Shijiazhuang 050016, China

${ }^{6}$ Department of Physics, Shandong University, Jinan 250100, China

${ }^{7}$ Institute of Modern Physics, SouthWest Jiaotong University, Chengdu 610031, China

${ }^{8}$ Faculty of Engineering, Kanagawa University, Yokohama 221-8686, Japan

${ }^{9}$ Faculty of Education, Utsunomiya University, Utsunomiya 321-8505, Japan

${ }^{10}$ Department of Physics, Konan University, Kobe 658-8501, Japan

${ }^{11}$ Research Institute for Science and Engineering, Waseda University, Tokyo 169-8555, Japan

${ }^{12}$ Faculty of Engineering, Yokohama National University, Yokohama 240-8501, Japan

${ }^{13}$ Department of Physics, Shinshu University, Matsumoto 390-8621, Japan

${ }^{14}$ Institute for Cosmic Ray Research, University of Tokyo, Kashiwa 277-8582, Japan

${ }^{15}$ National Center for Space Weather, China Meteorological Administration, Beijing 100081, China

${ }^{16}$ School of Information Science and Engineering, Shandong Agriculture University, Taian 271018, China

${ }^{17}$ Saitama University, Saitama 338-8570, Japan

${ }^{18}$ National Institute of Informatics, Tokyo 101-8430, Japan

${ }^{19}$ Sakushin Gakuin University, Utsunomiya 321-3295, Japan

${ }^{20}$ College of Science, China University of Petroleum, Qingdao, 266555, China

${ }^{21}$ Tokyo Metropolitan College of Industrial Technology, Tokyo 116-8523, Japan

${ }^{22}$ Max-Planck-Institut für Physik, München D-80805, Deutschland

${ }^{23}$ College of Industrial Technology, Nihon University, Narashino 275-8576, Japan

${ }^{24}$ Shonan Institute of Technology, Fujisawa 251-8511, Japan

${ }^{25}$ Japan Atomic Energy Agency, Tokai-mura 319-1195, Japan

${ }^{26}$ School of General Education, Shinshu University, Matsumoto 390-8621, Japan

E-mail: tibet.asgegmail.com

We plan to build a large (approximately 10,000 $\mathrm{m}^{* * 2}$ ) water Cherenkov- type muon detector array under the existing Tibet air shower array at 4,300 $\mathrm{m}$ above sea level, to observe 10-1000 $\mathrm{TeV}$ gamma rays from cosmic-ray accelerators in our Galaxy with wide field of view at very low background level. A gamma-ray induced air shower has significantly less muons compared with a cosmic-ray induced one. Therefore, we can effectively discriminate between primary gamma rays and cosmic-ray background events by means of counting number of muons in an air shower event by the muon detector array. We will make a progress report on the project, as some part of it started data-taking in 2014.

The 34th International Cosmic Ray Conference,

30 July- 6 August, 2015

The Hague, The Netherlands 


\section{Introduction}

The HESS group with 4 large IACTs in Namibia, reported on discovery of new 14 gammaray sources by galactic plane survey [W] in 2005. Surprisingly, most of them are UNIDentified (UNID) sources and faint in X-rays or other wavelengths. As the HESS survey was limited within the galactic plane in the southern hemisphere due to its narrow field of view, the importance of a wide field-of-view unbiased survey is emphasized. Furthemore, many of the 14 sources have a harder energy specturm (indices;-1.8 to -2.8) at TeV energies than the standard candle Crab (index;-2.6). The energy spectra turned out to extend up to approximately $10 \mathrm{TeV}$. Cosmic rays are supposed to be accelerated up to the knee energy region at supernova remnants (SNRs) in our galaxy. Therefore, we naturally expect gamma rays in the $100 \mathrm{TeV}$ region $(10-1000 \mathrm{TeV})$ which originate in $\pi^{0}$ decays produced by the accelerated cosmic rays interacting with matter surrounding the SNRs. The gamma ray emission of electron origin might be highly suppressed in the $100 \mathrm{TeV}$ region due to rapid decrease of inverse-Compton cross section by the Klein-Nishina effect as well as synchrotron radiation energy loss in the magnetic field around the SNRs. Multi-wavelength (radio, X-ray, gamma-ray) observations are essential to discriminate between the two processes.

\section{Tibet Air Shower Experiment}

The Tibet air shower array has been in operation at Yangbajing $\left(90^{\circ} 31^{\prime} \mathrm{E}, 30^{\circ} 06^{\prime} \mathrm{N} ; 4300 \mathrm{~m}\right.$ above sea level) in Tibet, China since 1990. It has continuously made a wide field-of-view (approximately 2 steradian) observation of cosmic rays and gamma rays in the northen sky.

The Tibet I array was constructed in 1990 [0], and it was gradually upgraded to the Tibet II by 1994 which consisted of 185 fast-timing (FT) scintillation counters placed on a $15 \mathrm{~m}$ square grid covering 36,900 $\mathrm{m}^{2}$, and 36 density (D) counters around the FT-counter array. Each counter has a plastic scintillator plate of $0.5 \mathrm{~m}^{2}$ in area and $3 \mathrm{~cm}$ in thickness. All the FT counters are equipped with a fast-timing 2-inch-diameter photomultiplier tube (FT-PMT), and 52 out of 185 FT counters are also equipped with a wide dynamic range 1.5-inch-diameter PMT (D-PMT) by which we measure up to 500 particles which saturates FT-PMT output, and all the D-counters have a DPMT. A $0.5 \mathrm{~cm}$ thick lead plate is put on the top of each counter in order to increase the counter sensitivity by converting gamma rays into electron-positron pairs in an electromagnetic shower. The mode energy of the triggered events in Tibet II is $\sim 10 \mathrm{TeV} / 7 \mathrm{TeV}$ for cosmic rays/gamma rays [3], 团].

In 1996, we added 77 FT counters with a $7.5 \mathrm{~m}$ lattice interval to a $5,200 \mathrm{~m}^{2}$ area inside the northern part of the Tibet II array. We called this high-density array Tibet HD [5]. The mode energy of the triggered events in Tibet $\mathrm{HD}$ is $\sim 3 \mathrm{TeV} / \sim 2 \mathrm{TeV}$ for cosmic rays/gamma rays. In the late fall of 1999, the array was further upgraded by adding 235 FT-counters to increase the high-density area from $5,200 \mathrm{~m}^{2}$ to $22,050 \mathrm{~m}^{2}$. We call this array and further upgraded one Tibet III [焑]. In 2002 , all of the $36,900 \mathrm{~m}^{2}$ area was

covered by the high-density array by adding 200 FT-counters. Finally we set up 56 FT-counters around the $36,900 \mathrm{~m}^{2}$ high density array and equipped 8 D-counters with FT-PMT in 2003. At present, the Tibet air shower array consists of 761 FT-counters ( 249 of which have a D-PMT) and 28 D-counters [प]. 
The performance of the Tibet air shower array has been well examined by observing the Moon's shadow (approximately 0.5 degree arc in diameter) in cosmic rays [ [6, [ [ ] $]$. The def icit map of cosmic rays around the Moon demonstrates the angular resolution to be around $0.9^{\circ}$ at a few $\mathrm{TeV}$ for the Tibet III array. The pointing error is estimated to be less than $\sim 0.01^{\circ}$ by displacement of the shadow's center from the apparent center in the north-south direction, as the east-west component of the geomagnetic field is very small at the experimental site. On the other hand, the shadow center displacement in the east-west direction due to the geomagnetic field enables us to spectroscopically estimate the energy scale uncertainty less than $\pm 12 \%$ [ [ ] ].

The first celestial $\mathrm{TeV}$ gamma-ray signal from the Crab (the standard candle in gamma-ra $\mathrm{y}$ astronomy) was detected by an imaging air Cherenkov telescope (IACT) developed by the Whipple group in 1989 [Q] $]$. The pioneering work opened a new energy window in astronomy.

Ten years later in 1999, we succeeded in observing multi-TeV gamma-ray signal from the Crab Nebula at $5.5 \sigma$ confidence level, using the Tibet HD array [G]. This was the first detection of multi$\mathrm{TeV}$ gamma-ray signal by a conventional air shower array. Subsequently, in 2000, we reported on detection of multi-TeV gamma rays successfully at 3.7 $\sigma$ level from Mrk501 which was in a highly flaring state during March 1997 and August 1997 [B]]. We also succeeded in observing multi-TeV gamma-ray flares at $5.1 \sigma$ level from Markarian 421 which was in a very active phase during the year 2000 and 2001 [目]. Multi-TeV gamma-ray signal was successfully detected from the Crab by the Tibet-III array as well [8].

We searched for $\mathrm{TeV}$ steady point sources in the northern sky. No statistically significant point source is found except for well established Crab and Mrk421 [ए0]].

Search for steady PeV gamma-ray emission from the Monogem ring region is done with the Tibet data taken from 1997 to 2004 [प]]. No evidence for statistically significant gamma-ray signal is found in the Monogem ring where the MAKET-ANI experiment recently claimed a positive detection of PeV high-energy cosmic radiation [ए]], although our flux sensitivity is approximately 10 times better than MAKET-ANI's.

The 2-dimensional anisotropy $(\sim \pm 0.2 \%)$ in the equatorial coordinates are obtained for the first time in the multi-TeV energy range [[13]]. Furthermore, we discovered a new diffuse cosmicray anisotropy (approximately $0.2 \%$ level excess) in the Cygnus region [14]], although we cannot judge currently whether it is caused by gamma rays and/or a local cosmic-ray anisotropy. Overlapping the large-scale anisotropy in the Cygnus region, a few spacially separated enhancements of smaller scale were observed. These small-scale excesses $\left(\sim 2^{\circ}\right)$ hint at the extended gamma-ray emission[44]. The two of the small-scale excesses were shortly established[प5], [ए6] as extended gamma-ray sources by the Milagro experiment capable of hadron/gamma-ray discrimination. Recently, we found a positive statistical correlation between the Fermi bright Galactic sources and our $\mathrm{TeV}$ gamma-ray excesses[[D]].

We also searched for multi-TeV diffuse gamma rays from the galactic plane [四, [8]]. As there was no significant signal, flux upper limits were obtained.

Meanwhile, the Milagro group recently claimed detection of $\mathrm{TeV}$ diffuse gamma-ray signal in the Cygnus region along the galactic plane [एप]. Currently, we can not draw any clear conclusion on the Milagro result, as the Tibet air shower array is unable to distinguish between cosmic gamma rays and hadrons in the multi-TeV energy region at present. 
To positively observe gamma rays in the $100 \mathrm{TeV}$ region with much better sensitivity than Tibet III, we plan to add a muon detector array to the air shower array. Gamma-ray induced electromagnetic air showers are muon-poor, while cosmic-ray induced hadronic ones are accompanied by many muons. This enables us to separate gamma rays from cosmic rays. Our current plan [20, [2], 22] relevant to gamma-ray astronomy above $10 \mathrm{TeV}$ is Tibet AS (Air Shower array with $83,000 \mathrm{~m}^{2}$ in area) + MD ( Muon Detector array with $\sim 10,000 \mathrm{~m}^{2}$ in area under Tibet AS). Each muon detector is a waterproof concrete pool, $7.2 \mathrm{~m}$ wide $\times 7.2 \mathrm{~m}$ long $\times 1.5 \mathrm{~m}$ deep in size, equipped with two 20 inch-in-diameter photomultiplier tubes (PMTs), i.e., HAMAMATSU R3600. The Tibet MD array are made up of 192 muon detectors set up $2.5 \mathrm{~m}$ underground. Its total effective area amounts approximately to $10,000 \mathrm{~m}^{2}$ for muons with energies more than $\sim 1 \mathrm{GeV}$. Our current MC simulation predicts that the cosmic-ray background events will be rejected by approximately 99.99\% at $100 \mathrm{TeV}$ using full-scale $\left(10,000 \mathrm{~m}^{2}\right) \mathrm{MD}$ array. the full-scale MD array will improve the sensitivity to gamma-ray sources by more than an order of magnitude. To confirm the hadron rejection power with this full-scale MD array, we constructed a prototype muon detector in 2007.

\section{Prototype Muon Detector}

In the late fall of 2007, we constructed a prototype water Cherenkov muon detector (approximately $100 \mathrm{~m}^{2}$ ) at $\sim 90 \mathrm{~m}$ away from the center of the existing Tibet airshower array.

The muon detector is made from reinforced concrete and composed of two water pool cells located at $2.5 \mathrm{~m}$ under the ground. Each cell is filled up with water of $1.5 \mathrm{~m}$ in depth, $7.2 \mathrm{~m} \times$ $7.2 \mathrm{~m}$ in area, equipped with three $20^{\prime \prime} \phi$ downward facing PMTs (HAMAMATSU R3600). Among the 3 PMTs, one is covered by a black sheet with $\sim 1 \%$ light transmission to effectively reduce the PMT gain (wider dynamic range), although we do not use it in this paper. The timing and charge information for each PMT is recorded by a trigger generated from surface scintillation counter array. We started the data taking in December, 2007.

\section{Results and Discussions}

As for the air shower MC simulation, air shower events are generated using CORSIKA code with QGSJET01c model for hadronic interactions assuming the cosmic-ray composition estimated mainly from direct observational data. Our MC simulation reproduces well the experimental data obtained by the prototype muon detector.

\section{Conclusions}

In 2007, we constructed a prototype water Cherenkov muon detector (approximately $100 \mathrm{~m}^{2}$ ) under the existing Tibet air shower array. We have been successfully taking data since December in 2007. The preliminary analysis shows that the data is in good agreement with our MC simulation . We are ready for the full-scale $\left(10,000 \mathrm{~m}^{2}\right)$ underground water Cherenkov muon detector array, $5 / 12$ of which was constructed now. We started data-taking by the $5 / 12$ scale muon detector with the Tibet air shower array in 2014. Figure $\square$ shows the expected integral sensitivity to a point 


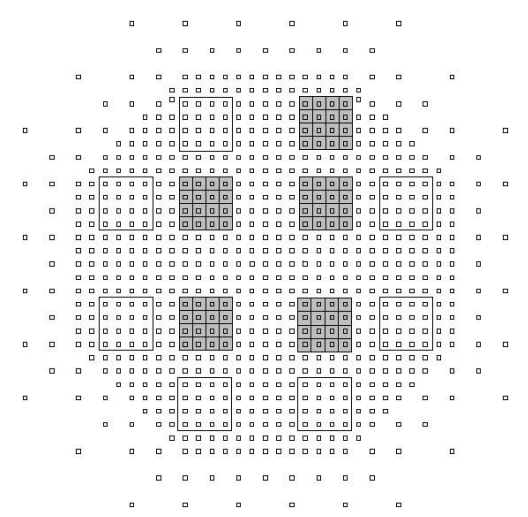

Figure 1: Schematic view of the Tibet MD. The 5/12 of the full-scale MD was completed and started data-taking in 2014.

gamma-ray source of the full-scale Tibet AS+MD project. As is seen in Fig. 凹, the Tibet AS+MD project gives the world-best sensitivity around $100 \mathrm{TeV}$.

\section{Acknowledgments}

The collaborative experiment of the Tibet Air Shower Arrays has been performed under the auspices of the Ministry of Science and Technology of China and the Ministry of Foreign Affairs of Japan. This work was supported in part by a Grant-in-Aid for Scientific Research on Priority Areas from the Ministry of Education, Culture, Sports, Science and Technology, by Grants-in-Aid for Science Research from the Japan Society for the Promotion of Science in Japan, and by the Grants from the National Nat ural Science Foundation of China and the Chinese Academy of Sciences.

\section{References}

[1] Aharonian, F.A. et al., Science 307, 1938 (2005).

[2] Amenomori, M. et al., Phys. Rev. Lett. 532, 2468 (1992).

[3] Amenomori, M. et al., Astrophys. J. 532, 302 (2000).

[4] Amenomori, M. et al., Astrophys. J. 580, 887 (2002).

[5] Amenomori, M. et al., Astrophy. J. 525, L93 (1999).

[6] Amenomori, M. et al., Astrophys. J. 598, 242 (2003).

[7] Amenomori, M. et al., Astrophys. J. 678, 1165 (2008).

[8] Amenomori, M. et al., Astrophys. J. 692, 61 (2009).

[9] Weeeks, T.C. et al., Astrophys. J. 342, 379 (1989).

[10] Amenomori, M. et al., Astrophys. J. 633, 1005 (2005).

[11] Amenomori, M. et al., Astrophys. J. 635, L53 (2005).

[12] Chilingarian, A. et al., Astrophys. J. 597, L129 (2003). 


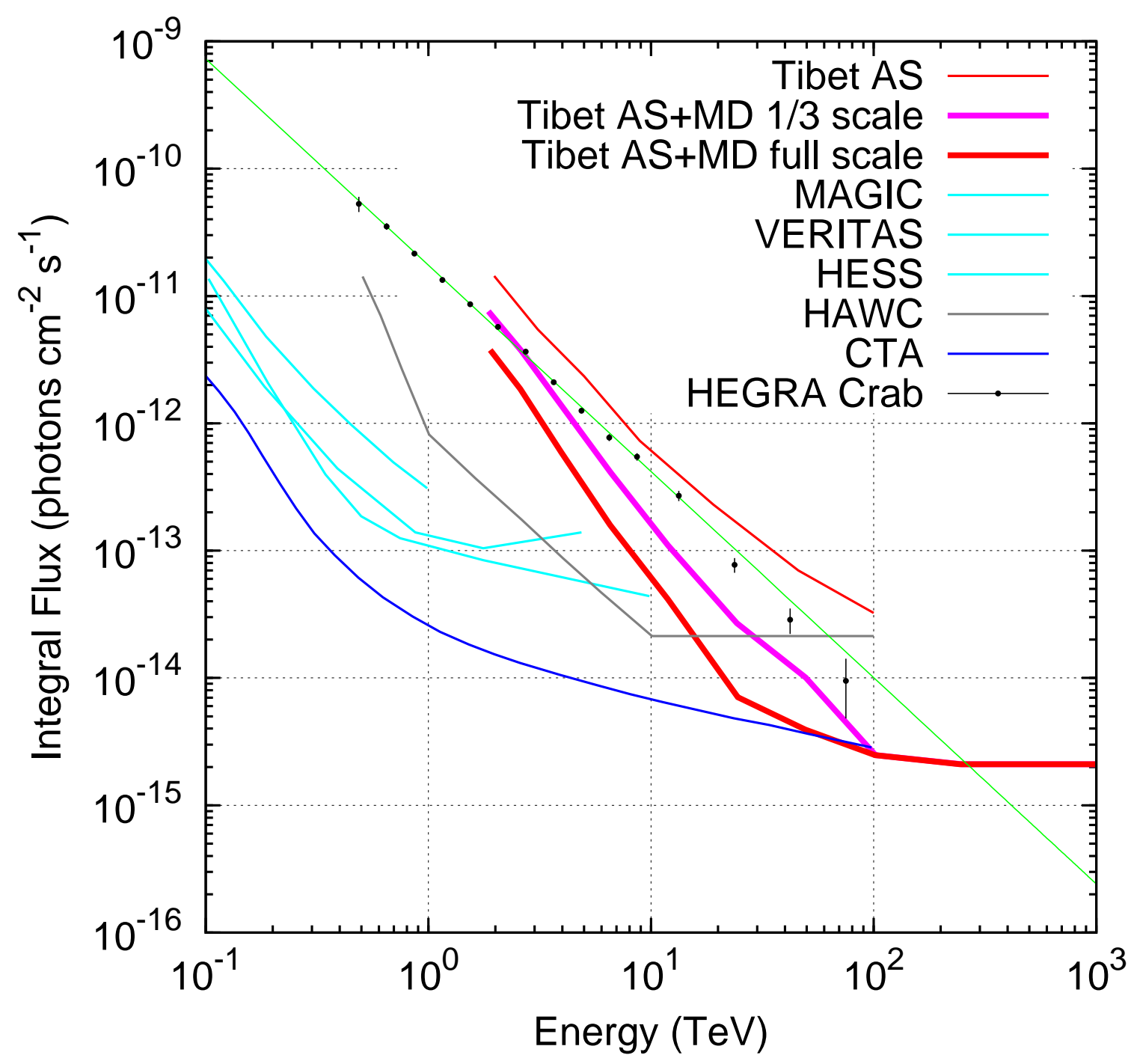

Figure 2: Expeced integral sensitivity to a gamma-ray point source of the Tibet AS+MD Project where AS area is assumed to be $37,000 \mathrm{~m}^{2}$ in this figure, together with various other experiments or projects.

[13] Amenomori, M. et al., Astrophys. J. 626, L29 (2005).

[14] Amenomori, M. et al., Science 314, 439 (2006).

[15] Abdo, A.A. et al., Astrophys. J. 658, 776 (2007).

[16] Abdo, A.A. et al., Astrophys. J. 664, L91 (2007).

[17] Amenomori, M. et al., Astrophys. J. 709, L6 (2010).

[18] Amenomori, M. et al., Advances in Space Research 37, 1932 (2006).

[19] Atkins, R. et al., Phys. Rev. Lett. 95, 251103 (2005).

[20] Amenomori, M. et al., AIP Conf. Proc. 1085, 723 (2008).

[21] Amenomori, M. et al., Journal of Physics: Conference Series 120, 062024 (2008).

[22] Sako, T.K. et al., Astropart. Phys. 32, 177 (2009). 